\title{
Characteristics of adverse drug reactions in a vemurafenib early post-marketing phase vigilance study in Japan
}

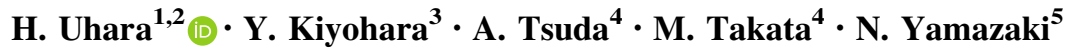

Received: 2 January 2017 / Accepted: 17 June 2017/Published online: 3 July 2017

(c) The Author(s) 2017. This article is an open access publication

\begin{abstract}
Background Post-approval research or monitoring is important to determine real-world safety of new products; however, evidence is scant for vemurafenib in Japanese patients. In Japan, a unique system is officially obligated to investigate post-approval safety. Here we report the first adverse drug reaction (ADR) data from vemurafenib-treated Japanese patients with metastatic melanoma. Data were collected in an early post-marketing phase vigilance (EPPV) study.

Methods ADRs were events for which a causal relationship with vemurafenib could not be ruled out or was unknown. ADR data were collected for patients treated with vemurafenib (960 mg bid) between 26 February and 25 August 2015.
\end{abstract}

Electronic supplementary material The online version of this article (doi:10.1007/s12094-017-1706-2) contains supplementary material, which is available to authorized users.

H. Uhara

uharah@sapmed.ac.jp

1 Department of Dermatology, Shinshu University School of Medicine, Nagano, Japan

2 Department of Dermatology, Sapporo Medical University School of Medicine, South 1, West 16, Chuo-ku, Sapporo, Hokkaido 060-8556, Japan

3 Dermatology Division, Shizuoka Cancer Center Hospital, Shizuoka, Japan

4 Chugai Pharmaceutical Co., Ltd., Tokyo, Japan

5 Department of Dermatologic Oncology, National Cancer Center Hospital, Tokyo, Japan
Results Among 95 patients, 46 patients had 118 ADRs (24 serious ADRs in 13 patients). The most common serious ADRs were hypersensitivity ( $n=1 ; 3$ events), arthralgia ( $n=2 ; 2$ events), pyrexia ( $n=2 ; 2$ events) and drug eruption ( $n=2 ; 2$ events). Seven patients had serious skin disorders or hypersensitivity, six of whom had prior antiprogrammed cell death-1 (PD-1) antibodies 5-35 days before starting vemurafenib. ADR reports of serious skin disorders appeared to be collected more rapidly than previously reported. Cutaneous squamous cell carcinoma developed in only one patient.

Conclusions EPPV in Japanese vemurafenib-treated patients identified no new safety signals. The most serious skin and hypersensitivity ADRs occurred in patients with prior anti-PD-1 exposure. Cutaneous squamous cell carcinoma appeared to be rare in Japanese patients. Further research is needed to clarify whether prior treatment with anti-PD-1 agents or racial differences affect the characteristic profile of cutaneous ADRs in Japanese patients.

Keywords Vemurafenib $\cdot$ Melanoma $\cdot$ Safety $\cdot$ Japanese patients

\section{Introduction}

Although melanoma is less common in Japan than in other developed countries, such as the US and Australia, the incidence of this condition has been increasing in recent years [1-3]. An estimated 1371 new cases were diagnosed in 2012 [4]; approximately $26-40 \%$ of these patients have the $B R A F^{\mathrm{V} 600}$ gene mutation $[5,6]$.

Before 2011, treatment options for patients with metastatic melanoma were limited and outcomes were poor [7]. In 2011, the US Food and Drug Administration approved 
the BRAF kinase inhibitor vemurafenib for the treatment of patients with metastatic melanoma harbouring the $B R A F^{\mathrm{V} 600}$ mutation [8]. This approval was based on the results of the phase III BRIM3 study in patients with previously untreated $B R A F^{\mathrm{V} 600 \mathrm{E}}$ mutation-positive metastatic or unresectable melanoma [9], which demonstrated significantly improved progression-free survival and overall survival in patients treated with vemurafenib compared with dacarbazine, the previous standard of care. Subsequently, other targeted agents have been approved for the treatment of patients with metastatic melanoma, including the MEK inhibitors, cobimetinib [10] and trametinib [11], the anti-cytotoxic T-lymphocyte antigen 4 (CTLA-4) inhibitor ipilimumab [12], and the anti-programmed cell death-1 (PD-1) agents nivolumab [13] and pembrolizumab [14].

Vemurafenib was approved for use in Japan in December 2014. The Japanese risk management plan includes an early post-marketing phase vigilance (EPPV) process, which is a unique system of post-marketing surveillance in Japan conducted during the 6 months after launch for most new active ingredients; similarly, approval of vemurafenib was conditional on performance of EPPV. During EPPV, medical representatives regularly visit medical institutions to collect safety information, in particular, to monitor the emergence of adverse drug reactions (ADRs). Here we report the findings from the vemurafenib EPPV study.

\section{Patients and methods}

\section{Study design}

Patients included in this prospective EPPV study were those who took part in the patient registry surveillance for vemurafenib that was conducted in Japan as a part of a post-approval commitment. All patients planning to use vemurafenib during the enrolment period were subject to registration in the study.

The EPPV study was planned to examine occurrences of ADRs, under the conditions of actual use, at medical institutions in Japan over the 6-month period following the launch of vemurafenib. During this period, medical representatives visited the study sites every 2 weeks and reported any ADRs either using an electronic ADR reporting form, by fax or email. The objective of the study was to provide information on the safety profile of vemurafenib and to detect any new safety signals appearing in real-world clinical practice.

The study was performed in accordance with Good Vigilance Practice of the Ministry of Health, Labour and Welfare, Japan. As this was a regulatory mandated study, patients were not required to provide informed consent; patients provided informed consent for treatment as per standard procedure at the individual institutions.

\section{Patients}

EPPV studies are not generally limited by the inclusion and exclusion criteria; however, patients with a baseline QTc $\geq 500 \mathrm{~ms}$ were not eligible for inclusion in line with the Japanese prescribing information for vemurafenib.

\section{Treatment}

Patients received vemurafenib at the usual adult dosage (960 mg bid), based on the approved dosage modified as needed in routine treatment as shown in Table 1 . Treatment continued as needed as EPPV studies do not have a predefined observational period. Treatment delays, suspensions, or dose modifications were performed as needed. Dose modifications were performed in cases of an adverse reaction or development of QT prolongation as shown in Table 1.

\section{Assessments}

Tests performed before treatment included $B R A F$ testing of tumour samples (cobas 4800 BRAFV600 Mutation Test, Roche Molecular Systems, Branchburg, NJ, USA), dermatological evaluation, head and neck examination, chest computed tomography, electrocardiogram, electrolyte measurement, liver enzyme levels and eye examination. During treatment, patients underwent the same tests routinely with the exception of $B R A F$ testing, which was only performed before starting administration of vemurafenib.

\section{Adverse drug reactions}

ADR data were collected as spontaneous reports during the 6-month EPPV period. Clinically significant ADRs were carefully monitored. ADRs were defined as events for which a causal relationship with vemurafenib could not be ruled out or was unknown. A serious ADR was defined as any event that resulted in death, was life-threatening, required inpatient hospitalisation or prolongation of existing hospitalisation for treatment, was equivalent in seriousness to any of the aforementioned events, resulted in persistent or significant disability/incapacity, or resulted in a congenital anomaly/birth defect. In the event of a serious ADR, more detailed information was collected on the patient using a case report form.

Labelled and unlabelled ADRs were collected. Labelled ADRs are those that might be expected based on the prescribing information. Unlabelled ADRs are those for which 
Table 1 Dose modification

\begin{tabular}{|c|c|}
\hline Grade $(\mathrm{NCI}-\mathrm{CTCAE})^{\mathrm{a}}$ & Recommended dose modification \\
\hline Grade 1 or Grade 2 (tolerable) & Dose modification unnecessary \\
\hline \multicolumn{2}{|l|}{ Grade 2 (intolerable) or Grade 3} \\
\hline 1st appearance & Interrupt treatment until recovery to Grade 0 to 1 or baseline, then resume dosing at $720 \mathrm{mg} \mathrm{bid}^{\mathrm{b}}$ \\
\hline 2nd appearance & Interrupt treatment until recovery to Grade 0 to 1 or baseline, then resume dosing at $480 \mathrm{mg}$ bid $^{\mathrm{c}}$ \\
\hline 3rd appearance & Discontinue permanently \\
\hline \multicolumn{2}{|l|}{ Grade 4} \\
\hline 1st appearance & $\begin{array}{l}\text { Discontinue permanently or, if it is preferable to continue treatment for a patient, interrupt } \\
\text { treatment until recovery to Grade } 0 \text { to } 1 \text { or baseline, then resume dosing at } 480 \mathrm{mg} \mathrm{bid}^{\mathrm{c}}\end{array}$ \\
\hline 2nd appearance & Discontinue permanently \\
\hline \multicolumn{2}{|l|}{ Prolongation of QT interval } \\
\hline $\begin{array}{l}\text { QTc }>500 \mathrm{~ms} \text { with }>60 \mathrm{~ms} \text { change from } \\
\text { pre-treatment values }\end{array}$ & Discontinue permanently \\
\hline \multicolumn{2}{|c|}{ QTc $>500 \mathrm{~ms}$ with $\leq 60 \mathrm{~ms}$ change from pre-treatment values } \\
\hline 1st appearance & Interrupt treatment until recovery to QTc $\leq 500 \mathrm{~ms}$, then resume treatment at $720 \mathrm{mg} \mathrm{bid}^{\mathrm{b}}$ \\
\hline 2nd appearance & Interrupt treatment until recovery to QTc $\leq 500 \mathrm{~ms}$, then resume treatment at $480 \mathrm{mg} \mathrm{bid}^{\mathrm{c}}$ \\
\hline 3rd appearance & Discontinue permanently \\
\hline
\end{tabular}

the nature or severity are not consistent with information in the relevant source document(s), such as the drug's prescribing information. Until source documents are amended, expedited reporting is required for additional occurrences of the reaction.

The risk management plan for vemurafenib investigated nine important identified risks: cutaneous squamous cell carcinoma (cuSCC), other secondary malignancies, liver injury, photosensitivity, corrected QT interval (QTc) prolongation, skin disorder (e.g. Stevens-Johnson syndrome, toxic epidermal necrolysis), hypersensitivity, eye disorders and potentiation of radiation injury. Four important potential risks were also investigated: progression of $R A S$ mutant malignancy, bone marrow depression, facial paralysis and gastrointestinal polyps. These were selected based on the results of clinical studies performed in Japan and elsewhere and events detected in post-marketing experience in countries outside of Japan where vemurafenib was approved.

\section{Results}

\section{Patient characteristics}

According to the patient registry surveillance, 95 patients were treated with vemurafenib in Japan during the EPPV period (26 February-25 August 2015). Of these, 52 patients were male and 43 patients were female; patient mean age was 58.9 years. Detailed information was only collected for patients who developed serious ADRs $(n=13$; Table 2).

At baseline, one patient had QT prolongation with a QTc value $<500 \mathrm{~ms}$. Five patients had electrolyte abnormalities that were adjusted before the start of the study and the patients were considered eligible.

\section{Safety}

The total number of ADRs collected during the EPPV period was 118 events in 46 patients. These are summarised in Table 3 and detailed in Supplementary Table 1. The most common ADRs were arthralgia, rash and pyrexia.

A total of 24 serious ADRs were reported in 13 patients. The most common were hypersensitivity (three events in one patient), and drug eruption, pyrexia and arthralgia (two events in two patients for each). Seven serious skin disorders including hypersensitivity reactions were reported, six of which were seen in the patients who had previously been treated with nivolumab or pembrolizumab within the 2 months before starting vemurafenib. Facial paralysis developed in one patient who had also been previously treated with nivolumab. These are summarised in Table 4. One patient with a history of nivolumab treatment experienced three hypersensitivity episodes after administration and re-administration of vemurafenib. The first episode occurred with myalgia, arthralgia, fatigue, and a fever of up to $38{ }^{\circ} \mathrm{C}$ and erythemas of various sizes across the patient's 
Table 2 Demographics and baseline characteristics of patients who developed serious adverse drug reactions $(n=13)$

\begin{tabular}{lc}
\hline Characteristic & Value \\
\hline Mean age, years (range) & $57.5(24-85)$ \\
Male, $n(\%)$ & $9(69)$ \\
ECOG performance status, $n(\%)$ & \\
0 & $7(54)$ \\
1 & $6(46)$ \\
2 & 0 \\
Metastatic melanoma stage, $n(\%)$ & \\
IIIc & $1(8)$ \\
IV & $1(8)$ \\
IV (M1b) & $2(15)$ \\
IV (M1c) & $9(69)$ \\
Treatment line, $n(\%)$ & \\
First & $1(8)$ \\
Second & $3(23)$ \\
Third & $6(46)$ \\
Fourth & $2(15)$ \\
Unknown & $1(8)$ \\
Prior therapy, $n(\%)$ & $1(8)$ \\
Interferon beta & $1(8)$ \\
Nivolumab & $1(8)$ \\
Dabrafenib & $1(8)$ \\
Pembrolizumab & \\
Unknown & \\
None & $1(8)$ \\
\hline
\end{tabular}

entire body 9 days after starting vemurafenib. A second onset of hypersensitivity developed $2 \mathrm{~h}$ after re-administration of vemurafenib, the symptoms of which included hyperemia, periocular skin disorder and mucosal symptoms, which spread systemically, with pyrexia of $39^{\circ} \mathrm{C}$. A third episode of hypersensitivity developed with rash (rose-pink erythema) on the patient's anterior chest 12 days after re-administration of a reduced dose of vemurafenib. Three episodes of hypersensitivities recurred during prednisolone taper and vemurafenib was discontinued.

Important identified risks and important potential risks are shown in Table 5. cuSCC, which is one of the most characteristic adverse events of BRAF inhibition, was observed in only one patent in the present study. All events of QTc prolongation that developed in this study were nonserious. One patient had a serious liver disorder (Grade 3). Vemurafenib was subsequently restarted at half the initial dose (480 mg bid) in this patient after improvement of the adverse event. However, vemurafenib was withdrawn for exacerbation of liver toxicity, which resolved 14 days after discontinuation of vemurafenib. One patient who developed a serious unlabelled ADR—hyperkalemia—had a
Table 3 Adverse drug reactions occurring in $>1$ patient in the Japanese early post-marketing phase vigilance period

\begin{tabular}{|c|c|c|c|}
\hline Event $(n)$ & Serious & Non-serious & All \\
\hline All events & 24 & 89 & 113 \\
\hline Arthralgia & 2 & 11 & 13 \\
\hline Rash & 1 & 7 & 8 \\
\hline Pyrexia & 2 & 6 & 8 \\
\hline Myalgia & 1 & 6 & 7 \\
\hline Drug eruption & 2 & 4 & 6 \\
\hline Photosensitivity reaction & & 5 & 5 \\
\hline Skin disorder & 1 & 3 & 4 \\
\hline Hypersensitivity & 3 & & 3 \\
\hline Alopecia & & 3 & 3 \\
\hline Erythema multiforme & 1 & 2 & 3 \\
\hline PPE & & 3 & 3 \\
\hline Decreased appetite & & 2 & 2 \\
\hline Bundle branch block right & & 2 & 2 \\
\hline Diarrhoea & & 2 & 2 \\
\hline Hepatic function abnormal & & 2 & 2 \\
\hline Acne & & 2 & 2 \\
\hline Erythema nodosum & & 2 & 2 \\
\hline Hyperkeratosis & & 2 & 2 \\
\hline Malaise & & 2 & 2 \\
\hline QT prolonged & & 2 & 2 \\
\hline Neutrophil count decreased & 1 & 1 & 2 \\
\hline Platelet count decreased & & 2 & 2 \\
\hline
\end{tabular}

$P P E$ palmar-plantar erythrodysesthesia syndrome

sudden increase in potassium level after vemurafenib administration that was controlled with a potassium chelating agent. This event was followed by tumour regression.

\section{Discussion}

Since the approval of vemurafenib for the treatment of patients with metastatic melanoma in 2011, a large body of evidence has been accumulated demonstrating the tolerability of vemurafenib. However, the data are limited to the clinical trial setting and to Caucasian patients. Little evidence exists in real-world use, although post-approval research or monitoring is important in determining the realworld safety of new products. Moreover, evidence of the real-world safety of vemurafenib is scant in Japanese patients. In Japan, there is a unique system (EPPV studies) that is officially obligated to investigate post-approval safety. After the approval of vemurafenib in Japan in 2015, the present EPPV study was performed in line with Japanese regulatory requirements. The aims of this study were 
Table 4 Serious skin disorders and hypersensitivity reactions occurring in patients in the vemurafenib early post-marketing phase vigilance study

\begin{tabular}{lllll}
\hline Case no. & Events & Prior therapy & Outcome & Re-administration of vemurafenib \\
\hline 1 & Skin disorder & Pembrolizumab until Day -28 & Recovered & Yes \\
2 & Hypersensitivity & Nivolumab until Day -5 & Improved & Yes \\
3 & Rash & Interferon beta & Recovered & Yes \\
4 & Drug eruption & Nivolumab until Day -21 & Improved & Yes \\
5 & Stevens-Johnson syndrome & Nivolumab until Day -21 & Improved & No \\
6 & Erythema multiforme & Nivolumab until Day -35 & Recovered & Yes \\
7 & Drug eruption & Nivolumab until Day -35 & Recovered & No \\
\hline
\end{tabular}

${ }^{a}$ Previously reported by Imafuku et al. [15]. This patient experienced three hypersensitivity episodes

Table 5 Important identified risks

\begin{tabular}{|c|c|c|c|c|}
\hline \multirow[t]{2}{*}{ Adverse drug reaction } & \multicolumn{2}{|l|}{ Serious events } & \multicolumn{2}{|l|}{ All events } \\
\hline & No. of patients & No. of events & No. of patients & No. of events ${ }^{a}$ \\
\hline \multicolumn{5}{|l|}{ Important identified risks } \\
\hline cuSCC & 1 & 1 & 1 & 1 \\
\hline Other second malignancy & 0 & 0 & 0 & 0 \\
\hline Liver injury & 1 & 1 & 5 & 5 \\
\hline Photosensitivity & 0 & 0 & 5 & 5 \\
\hline QTc prolongation & 0 & 0 & 2 & 2 \\
\hline Skin disorder & 5 & 5 & 17 & 17 \\
\hline Hypersensitivity & 2 & 4 & 10 & 12 \\
\hline Eye disorders & 0 & 0 & 2 & 2 \\
\hline \multicolumn{5}{|l|}{ Important potential risks } \\
\hline Progression of $R A S$ mutant malignancy & 0 & 0 & 0 & 0 \\
\hline Facial paralysis & 1 & 1 & 1 & 1 \\
\hline Myelosuppression & 1 & 3 & 3 & 5 \\
\hline Gastrointestinal polyps & 0 & 0 & 0 & 0 \\
\hline
\end{tabular}

cuSCC cutaneous squamous cell carcinoma

${ }^{a}$ Serious and non-serious events

to clarify the real-world safety of vemurafenib in Japanese patients and to assess whether racial differences exist between Caucasian and Japanese patients. This EPPV study identified no new safety signals for vemurafenib compared with previous reports from large-scale clinical studies $[9,16]$, although some differences in cutaneous ADRs were observed in our Japanese patients.

The most common ADRs observed in Japanese patients in the EPPV study were arthralgia and rash. Serious ADRs included hypersensitivity, arthralgia, pyrexia and drug eruption. These were similar to previous reports $[9,16]$. However, cuSCC was seen in only one patient and keratoacanthoma was not observed in our study. The previous safety study reported that cuSCC, keratoacanthoma and Bowen's disease developed in $14 \%$ of patients [16]. Because the number of the examined cases is limited in the present study, further examination is required to clarify whether difference in skin type influences the occurrence of vemurafenib-related skin tumours. It is also possible that duration of treatment may be a risk factor in the development of drug-related skin tumours; further investigation of this is also warranted.

Skin reactions were common in patients in this study and included rash, drug eruption, photosensitivity and erythema multiforme in line with previously reported clinical trials $[9,16]$.

Six of seven patients in the EPPV study who developed serious skin disorders had previously been treated with nivolumab or another PD-1 antibody before receiving vemurafenib. Nivolumab was approved before vemurafenib in Japan, resulting in many patients using nivolumab first, before starting vemurafenib after its approval in February 2015. This is not a comparative study, but these results may suggest that previous treatment with anti-PD-1 
antibodies could affect the seriousness of skin disorders if vemurafenib is used after anti-PD-1 agents. Further research is needed to clarify whether prior treatment with anti-PD-1 agents induces more severe skin disorders including hypersensitivity ADRs. The results of the EPPV study may help assess the safety characteristics of vemurafenib when used after anti-PD-1 antibodies.

Other important serious AEs in our study included facial paralysis in one patient. Facial paralysis associated with vemurafenib has been previously reported [17-20]. The patient who developed facial paralysis in our study had previous treatment history of nivolumab and concurrently developed serious muscular weakness, drug eruption (causality of vemurafenib was denied) and non-serious photosensitivity reaction. Because the number of such cases is limited in the present study, it is unclear whether these events were common in Japanese patients.

Some limitations of the present study should be considered. Detailed information was only collected for patients in whom serious ADRs developed. In addition, the observation period was short, which may have limited the number of ADRs observed. Further observation over a longer period may be needed to clarify the safety profile of vemurafenib in Japanese patients.

In conclusion, this EPPV study identified no new safety signals for vemurafenib in Japanese patients with metastatic melanoma; however, some differences compared with previous studies were observed, namely a lower incidence of cuSCC and keratoacanthoma, and higher incidence of serious skin disorders. The most serious skin and hypersensitivity ADRs occurred in patients with prior anti-PD-1 exposure. Further research is needed to clarify whether prior treatment with anti-PD-1 agents or racial differences affect cutaneous ADRs in Japanese patients.

Acknowledgements This work was sponsored by Chugai Pharmaceutical Co., Ltd. Medical writing/editing support was provided by Miller Medical Communications and was funded by Chugai Pharmaceutical Co., Ltd.

\section{Compliance with ethical standards}

Conflict of interest Hisashi Uhara, Yoshio Kiyohara and Naoya Yamazaki are members of the Vemurafenib Appropriate Use Committee for Chugai Pharmaceutical Co. Ltd, and have received funding for medical research from Chugai Pharmaceutical Co. Ltd. Hisashi Uhara has received honoraria from Chugai, Ono Pharmaceutical, Bristol-Myers Squibb Japan, Merck Sharp \& Dohme and Mochida Pharmaceutical; has acted as a consultant for Chugai, Ono Pharmaceutical, Bristol-Myers Squibb Japan, Merck Sharp \& Dohme and Novartis; and has been part of speakers' bureaux for Chugai, Ono Pharmaceutical, Bristol-Myers Squibb Japan and Mochida Pharmaceutical. Yoshio Kiyohara has received honoraria from Chugai, Ono Pharmaceutical, Bristol-Myers Squibb Japan, Merck Sharp \& Dohme, Novartis, GlaxoSmithKline, Merck Serono, AstraZeneca Japan and Takeda; has acted as a consultant for Chugai, Ono Pharmaceutical, Bristol-Myers Squibb Japan, Merck Sharp \& Dohme, Novartis and
GlaxoSmithKline; and has been part of speakers' bureaux for Chugai, Ono Pharmaceutical, and Bristol-Myers Squibb Japan. Naoya Yamazaki has received honoraria from and been part of speakers' bureaux for Chugai, Takeda, Ono Pharmaceutical, Bristol-Myers Squibb Japan, Maruho, Merck Serono, Lilly Japan, Yakult, Boehringer Ingelheim, Bayer, GlaxoSmithKline, Kissei Pharmaceuticals, Mitsubishi Tanabe Pharma and AstraZeneca Japan; and has received honoraria from Chugai, Ono Pharmaceuticals, Bristol-Myers Squibb Japan and Bayer. Asako Tsuda and Mariko Takata are in full-time employment with Chugai Pharmaceutical Co., Ltd.

Ethical approval The study was performed in accordance with Good Vigilance Practice of the Ministry of Health, Labour and Welfare, Japan.

Informed consent As this was a regulatory mandated study, patients were not required to provide informed consent; patients provided informed consent for treatment as per standard procedure at the individual institutions.

Open Access This article is distributed under the terms of the Creative Commons Attribution 4.0 International License (http://crea tivecommons.org/licenses/by/4.0/), which permits unrestricted use, distribution, and reproduction in any medium, provided you give appropriate credit to the original author(s) and the source, provide a link to the Creative Commons license, and indicate if changes were made.

\section{References}

1. Ishihara K, Saida T, Yamamoto A, Japanese Skin Cancer Society Prognosis and Statistical Investigation Committee. Updated statistical data for malignant melanoma in Japan. Int J Clin Oncol. 2001;6:109-16.

2. Ishihara K, Saida T, Otsuka F, Yamazaki N, Prognosis and Statistical Investigation Committee of the Japanese Skin Cancer Society. Statistical profiles of malignant melanoma and other skin cancers in Japan: 2007 update. Int J Clin Oncol. 2008;13:33-41.

3. Katanoda K, Hori M, Matsuda T, Shibata A, Nishino Y, Hattori M, et al. An updated report on the trends in cancer incidence and mortality in Japan, 1958-2013. Jpn J Clin Oncol. 2015;45:390-401.

4. Globocan 2012: Estimated cancer incidence, mortality and prevalence worldwide in 2012. Japan. Available at http://globocan.iarc.fr/Pages/fact_sheets_ population.aspx. Accessed December 22, 2016.

5. Sakaizawa K, Ashida A, Uchiyama A, Ito T, Fujisawa Y, Ogata D, et al. Clinical characteristics associated with BRAF, NRAS and KIT mutations in Japanese melanoma patients. J Dermatol Sci. 2015;80:33-7.

6. Yamazaki N, Tanaka R, Tsutsumida A, Namikawa K, Eguchi H, Omata W, et al. BRAF V600 mutations and pathological features in Japanese melanoma patients. Melanoma Res. 2015;25:9-14.

7. Mouawad R, Sebert M, Michels J, Bloch J, Spano JP, Khayat D. Treatment for metastatic malignant melanoma: old drugs and new strategies. Crit Rev Oncol Hematol. 2010;74:27-39.

8. Kim G, McKee AE, Ning YM, Hazarika M, Theoret M, Johnson JR, et al. FDA approval summary: vemurafenib for treatment of unresectable or metastatic melanoma with the BRAFV600E mutation. Clin Cancer Res. 2014;20:4994-5000.

9. Chapman PB, Hauschild A, Robert C, Haanen JB, Ascierto P, Larkin J, et al. Improved survival with vemurafenib in melanoma with BRAF V600E mutation. N Engl J Med. 2011;364:2507-16.

10. Larkin J, Ascierto PA, Dréno B, Atkinson V, Liszkay G, Maio M, et al. Combined vemurafenib and cobimetinib in BRAF-mutated melanoma. N Engl J Med. 2014;371:1867-76.

11. Flaherty KT, Infante JR, Daud A, Gonzalez R, Kefford RF, Sosman J, et al Combined BRAF and MEK inhibition in melanoma with BRAF V600 mutations. N Engl J Med. 2012;367:1694-703.

12. Hodi FS, O'Day SJ, McDermott DF, Weber RW, Sosman JA, Haanen JB, et al. Improved survival with ipilimumab in patients with metastatic melanoma. N Engl J Med. 2010;363:711-23.

13. Robert C, Long GV, Brady B, Dutriaux C, Maio M, Mortier L, et al. Nivolumab in previously untreated melanoma without BRAF mutation. N Engl $\mathrm{J}$ Med. 2015;372:320-30. 
14. Robert C, Schachter J, Long GV, Arance A, Grob JJ, Mortier L, et al. Pembrolizumab versus ipilimumab in advanced melanoma. $\mathrm{N}$ Engl $\mathrm{J}$ Med. 2015;372:2521-32.

15. Imafuku K, Yoshino K, Ishiwata K, Otobe S, Tsuboi S, Ohara K, Hata H. Severe rash associated with vemurafenib administration following nivolumab therapy. J Eur Acad Dermatol Venereol. 2016;30:e84-6.

16. Larkin J, Del Vecchio M, Ascierto PA, Krajsova I, Schachter J, Neyns B, et al. Vemurafenib in patients with BRAF(V600) mutated metastatic melanoma: an open-label, multicentre, safety study. Lancet Oncol. 2014;15:436-44.

17. Shailesh FN, Singh M, Tiwari U, Hutchins LF. Vemurafenib-induced bilateral facial palsy. J Postgrad Med. 2014;60:187-8.
18. Klein O, Ribas A, Chmielowski B, Walker G, Clements A, Long GV, Kefford RF. Facial palsy as a side effect of vemurafenib treatment in patients with metastatic melanoma. J Clin Oncol. 2013;31:e215-7.

19. Sosman JA, Kim KB, Schuchter L, Gonzalez R, Pavlick AC, Weber JS, et al Survival in BRAF V600-mutant advanced melanoma treated with vemurafenib. N Engl J Med. 2012;366:707-14.

20. McArthur GA, Chapman PB, Robert C, Larkin J, Haanen JB, Dummer R, et al. Safety and efficacy of vemurafenib in BRAF(V600E) and BRAF(V600K) mutation-positive melanoma (BRIM-3): extended follow-up of a phase 3, randomised, open-label study. Lancet Oncol. 2014;15:323-32. 